\title{
Lumen
}

Selected Proceedings from the Canadian Society for Eighteenth-Century Studies

\section{La Lettre sur les sourds et muets (1751) de Denis Diderot : une rhétorique du punctum temporis}

\section{Marc-André Bernier}

Volume 18, 1999

Representations of Time in the XVIIIth Century

Le temps et ses représentations au dix-huitième siècle

URI : https://id.erudit.org/iderudit/1012363ar

DOI : https://doi.org/10.7202/1012363ar

Aller au sommaire du numéro

Éditeur(s)

Canadian Society for Eighteenth-Century Studies / Société canadienne d'étude du dix-huitième siècle

ISSN

1209-3696 (imprimé)

1927-8284 (numérique)

Découvrir la revue

Citer cet article

Bernier, M.-A. (1999). La Lettre sur les sourds et muets (1751) de Denis Diderot : une rhétorique du punctum temporis. Lumen, 18, 1-11.

https://doi.org/10.7202/1012363ar

Copyright (C Canadian Society for Eighteenth-Century Studies / Sociéte canadienne d'étude du dix-huitième siècle, 1999
Ce document est protégé par la loi sur le droit d'auteur. L'utilisation des services d'Érudit (y compris la reproduction) est assujettie à sa politique d'utilisation que vous pouvez consulter en ligne.

https://apropos.erudit.org/fr/usagers/politique-dutilisation/ 


\section{La Lettre sur les sourds et muets (1751) de Denis Diderot: une rhétorique du punctum temporis}

Quiconque a fréquenté les ouvrages de rhétorique du XVIIIe siècle sait que la tradition oratoire à laquelle se rattache chacun d'entre eux se signale par le rappel imperturbable d'un fonds commun d'exemples. Cette première observation, la Lettre sur les sourds et muets que Denis Diderot fait paraître en 1751 l'illustre à plus d'un titre. La plupart des exemples qu'on y retrouve provient moins, comme l'a souvent relevé la critique actuelle, d'une relecture personnelle des textes, que d'ouvrages de rhétorique et de poétique parus au cours de la première moitié du siècle. ${ }^{1}$ De ce nombre, il en est un sur lequel Diderot s'attarde plus volontiers: il s'agit d'un vers tiré du Lutrin de Boileau et qui 'peint' en ces termes la figure allégorique de la Mollesse, laquelle '[s]oupire, étend les bras, ferme l'œil et s'endort. ${ }^{2}$

$S^{\prime}$ il est vrai que ce vers de Boileau avait déjà fait l'objet de nombreux commentaires pendant la première moitié du XVIIIe siècle, depuis la Manière d'enseigner et d'étudier les belles-lettres (1726-1728) de Charles Rollin jusqu'à la Poétique françoise (1736) de l'abbé d'Olivet, on doit surtout observer, à la suite de Diderot, 'que le poète a quatre actions à peindre [...] et qu'en les renfermant toutes quatre dans un seul vers, le poète a satisfait à la promptitude avec laquelle elles ont coutume de se succéder' (LSM, 170).

Autrement dit, 'peindre' la succession dans le temps de ces quatre actions réclame du poète de la 'promptitude', de sorte qu'avec cette peinture d'une pluralité de moments distincts s'alliant à une exigence de brièveté dans leur rendu se trouve mise en cause une certaine conception du temps propre à l'expérience vécue.

$C^{\prime}$ est ce rapport complexe entre temporalité et concision d'une forme oratoire dont je souhaite proposer l'examen. A cette fin, il faudra d'abord reprendre le problème de l'inversion sur lequel s'ouvre la Lettre, de manière à rappeler le contexte d'un débat où le recours au personnage du sourd et muet va servir à mettre en évidence un moment fondateur: 
celui où la multitude des sensations, des idées et des mots se présente confusément à l'esprit dans un 'instant unique.' On indiquera ensuite dans quelle mesure cette enquête linguistique et épistémologique parvient à établir sur les données immédiates de l'expérience sensible une théorie de l'éloquence devenue indissociable du rendu de cet 'instant unique' ou, pour mieux dire, de ce punctum temporis. On voudra bien considérer enfin à quel point cette réflexion sur la temporalité met Diderot à même de ressaisir et de réinventer tout à la fois certaines des notions essentielles de la rhétorique du XVIIIe siècle.

Adressée à l'abbé Batteux, l'auteur bien connu des Beaux arts réduits à un même principe (1746), la Lettre sur les sourds et muets se donne à lire comme un texte qui entend adopter le ton libre d'une conversation où la pensée se déploie, suivant l'expression de Diderot, en 'voltigeant' (LSM, 133) d'un objet à un autre. Le sous-titre de la Lettre se charge d'indiquer le premier de ces objets: la question de l'inversion, problème en apparence incident, mais sur lequel s'attarde toute la première partie de l'ouvrage. Il n'y a pas lieu d'insister ici sur ce problème qu'avait formulé, dès 1667, Louis Le Laboureur, dans une lettre dont l'ambition consistait à établir les Avantages de la langue françoise sur la langue latine en montrant qu'en français 'les mots tiennent entr'eux l'ordre que la raison leur donne', ordre au demeurant 'plus naturel à l'esprit, et plus conforme au bon sens. ${ }^{3}$ Les travaux de Bernard Tocane ou encore d'Ulrich Ricken ont déjà mis en évidence l'importance et les circonstances particulières d'un tel débat à l'occasion duquel, vers le milieu du XVIIIe siècle, va venir s'opposer la tradition rationaliste issue de Port-Royal et illustrée par Dumarsais, aux thèses du sensualisme défendues par Condillac et l'abbé Batteux. ${ }^{4}$ Suivant les uns, l'inversion se définit, et c'est là une idée chère à Dumarsais, comme un renversement contrevenant à 'l'ordre de la syntaxe des vues de l'esprit', ordre réputé naturel et assimilé à la séquence logique sujet/verbe/complément de la phrase française:

Si Virgile a dit virum, précise Dumarsais, c'est que, selon l'ordre de la syntaxe des vues de l'esprit, virum est après cano; ainsi quoique selon la construction élégante et usuelle, qui admet presque toujours l'inversion en latin, Virgile ait dit arma virumque cano, il avoit eu nécessairement dans l'esprit, par une priorité $\mathrm{d}$ 'ordre, cano avant arma virumque; telle est la suite des vues de l'esprit [...] et ce n'est jamais que relativement à cette suite qu'il y a inversion. ${ }^{5}$

Pour les autres, et cette réflexion est de Batteux, 'l'arrangement naturel des mots' est plutôt 'prescrit par l'intérêt ou le point de vue de celui qui parle' - autrement dit, par l'ordre suivant lequel se succèdent les sensations dans l'esprit. ${ }^{6}$ 
C'est dans ce contexte que la Lettre sur les sourds et muets va reprendre la question des inversions, s'inspirant tantôt de Dumarsais et tantôt de l'abbé Batteux jusque dans le choix de ses exemples. ${ }^{7}$ Seulement, la conception duelle de l'ordre autour de laquelle s'articulait jusqu'alors le débat sera, en quelque sorte, surmontée à la faveur d'une tripartition du problème. Il importe de distinguer, précise Diderot, entre un 'ordre didactique des idées', assimilable à la logique toute géométrique des 'vues de l'esprit', puis un 'ordre d'institution', qui correspond à la syntaxe particulière de chaque langue, et, enfin, un 'ordre $d$ 'invention des mots' $(L S M, 164)$, lui-même fondé sur la multitude confuse et simultanée des sensations, des idées et des mots - et susceptible, à ce titre, de renverser, voire de ruiner, toute la problématique de l'inversion. ${ }^{8}$

Sur les deux premiers types d'ordre que la Lettre évoque, on ne fera, suivant le mot de Diderot, que 'voltiger': ce sont moins les considérations relatives à l'ordre didactique ou d'institution que les réflexions touchant l'ordre d'invention des mots qui sollicitent la question de la représentation de l'expérience vécue du temps. Que l'on songe néanmoins que, si $1^{\prime \prime}$ ordre didactique' se confond chez Diderot avec une sorte d'organisation géométrisée de la phrase, cet ordre dont le principe est universel ne s'affirme pourtant qu'au terme de la formation des langues. Ainsi cette question conduit-elle naturellement à l'ébauche d'une linguistique comparative qui place le français à la pointe extrême du devenir historique de la rationalité. A la différence de l'ordre d'institution régissant la syntaxe de toutes les autres langues, le français suit de si près la marche didactique de l'esprit qu'il s'est entièrement dégagé de 'la balbutie des premiers âges', ordonnant désormais les choses 'comme l'esprit est forcé de les considérer dans quelque langue qu'on écrive' (LSM, 164-65). ${ }^{9}$ Langue 'plus propre aux sciences', langue que 'le bon sens choisirait' (LSM, 165), le français ne saurait pourtant faire valoir ces titres pour revendiquer le privilège de se fonder sur un ordre prétendu 'naturel' des idées, comme avait déjà pu le soutenir le rationalisme d'un Dumarsais assimilant ordre logique et didactique à une sorte d'ordo naturalis. Comme l'a déjà fait observer Ulrich Ricken,

Diderots Beachtung der historischen Perspektive führt ihn bis zu der genial anmutenden These [...] An Stelle von Kategorien der zeitlosen raison sind Kategorien der aus der sensation hervorgegangen réflexion gesetz worden. ${ }^{10}$

Certes, 'la formation des langues exigeait la décomposition' (LSM, 162) logique et analytique de la pensée qui, parvenue au dernier terme de son évolution, se confond avec la marche didactique de la syntaxe française, mais, ajoute enfin Diderot, cet ordre n'est pas celui de la 'nature.' 
Une fois parvenue à ce point, la Lettre va chercher à interroger cette 'nature' et, comme il s'agit de mettre en lumière 'les véritables notions de la formation du langage' (LSM, 142), tout invite le sensualisme de Diderot à en appeler aux données immédiates de l'expérience sensible. C'est alors que commence le récit des observations effectuées sur un sourd et muet, personnage destiné à faire paraître sous les dehors et la figure de l'expérience une réflexion qui, centrée sur le sentiment intime de la temporalité, va chercher à mettre en évidence un moment fondateur: celui où la multitude des sensations, des idées et des expressions s'offre pêle-mêle à l'esprit dans un instant unique. Un premier exemple va permettre de mieux comprendre ce que met en cause cette simultanéité confuse des sensations à laquelle songe Diderot et qui régnerait dans la pensée avant que la langue ne la décompose en idées distinctes appelées ensuite à s'ordonner dans le discours. A s'entretenir avec un sourd et muet de naissance, raconte la Lettre, une singularité frappe aussitôt l'attention et se transforme bientôt en 'une difficulté presque insurmontable': l'embarras où l'on se trouve de faire sentir à son interlocuteur les différences que nous mettons entre les temps du verbe. 'On est jamais sûr', poursuit Diderot, 'de lui avoir fait entendre la différence des temps je fis, $j$ 'ai fait, je faisais, $j$ 'aurais fait': on pourrait même conjecturer de cela que 'les signes des temps ou des portions de la durée ont été les derniers inventés' dans les langues (LSM, 152). La perception du temps ne forme dans l'esprit qu'une seule vue de l'âme' (LSM, 158), tableau unique que le discours décompose par la suite suivant l'ordre de succession des termes, les mots exprimant, en quelque sorte, les divers 'modes d'une seule sensation' (LSM, 158). Comparez le langage des gestes d'un sourd au langage articulé et il sera constant que 'l'état de l'âme dans un instant indivisible fut représenté par une foule de termes que la précision du langage exigea, et qui distribuèrent une impression totale en parties' (LSM, 161). En somme,

notre âme est un tableau mouvant d'après lequel nous peignons sans cesse [...] La formation des langues exigeait la décomposition; mais voir un objet, le juger beau, éprouver une sensation agréable, désirer la possession, c'est l'état de l'âme dans un même instant. (LSM, 161-62)

Que conclure de cette 'âme' assimilée à un 'tableau mouvant', si ce n'est qu'à la simultanéité des sensations doit répondre, dans l'esprit, celle des idées et que, du point de vue de ce 'moment indivisible de l'âme', il devient fort difficile, voire impossible, de déterminer quel pourrait être l'ordre naturel du discours?

C'est afin d'illustrer cette hypothèse d'une âme susceptible de s'occuper en même temps de plusieurs objets distincts que la Lettre recourt aux 
métaphores, si fameuses et si souvent commentées, du 'clavecin oculaire' ou de l' homme horloge.' L'argument essentiel que renferment ces figures se réduit à supposer une analogie: de même qu'il est possible de lier les sons ou les couleurs de manière à former des accords, de même voit-on le discours attacher 'plusieurs idées à une seule expression.' Faute d'avoir 'vingt bouches' pour rendre d'un même coup de glotte des impressions multiples qui n'ont pas 'dans l'âme ce développement successif du discours', la langue trouve dans une elocutio serrée et rapide une ressource propre à suppléer à cette difficulté en représentant à la faveur d'un seul trait oratoire la vivacité tumultueuse des sentations qui se pressent dans l'âme. Dès lors, conclut Diderot, discours, elocutio et figures de style découvrent à l'analyse le ressort essentiel de leur éloquence ou, pour mieux dire, de leur 'énergie' (LSM, 158). Issues tantôt de la rhétorique et tantôt de l'épistémologie sensualiste, toutes ces idées forment, en s'unissant l'une à l'autre, une configuration dont la complexité même exige qu'on en interroge davantage les détours. Autrement dit, quel sens doit-on assigner à une conception de la temporalité où passé, présent et futur s'abolissent dans l'instant unique de la sensation, à partir du moment où celle-ci s'allie à une réflexion sur la rapidité et sur l'énergie de l'élocution oratoire?

Si l'on en juge par la plupart des auteurs qui ont traité de rhétorique dans la première moitié du XVIIIe siècle, qu'il s'agisse de Claude Buffier ou de Charles Porée, de l'abbé Goujet ou de l'abbé d'Olivet, on s'aperçoit qu'une même observation revient sans cesse et avec insistance. A chaque fois, lit-on, le goût du siècle en matière d'éloquence semble se déclarer en faveur de ce qu'on pourrait appeler, suivant le mot de Goujet, 'une rapide simplicité.'11 'Que voulez-vous?', résume Charles Porée, 'nous aimons la briéveté. Se mêle-t'on de nous instruire; nous voulons qu'on nous dise beaucoup en peu de mots. ${ }^{12}$ Surtout, la plupart des textes consacrés à la rhétorique ne manquent pas de rappeler que, dans cette 'briéveté' et cette 'rapide simplicité' propres à l'éloquence moderne, entre sans cesse le souvenir de Sénèque le Philosophe. Les témoignages sont ici innombrables. C'est tantôt Porée, pour qui 'le stile coupé [...] à la Sénèque $[\ldots]$ paroît $[\ldots]$ le plus propre à aiguiser l'esprit des jeunes gens $^{\prime \prime}{ }^{13}$ tantôt l'abbé d'Olivet se déclarant hostile à tous les jeunes auteurs de son siècle qui 'se plaisent à marcher dans une nouvelle route, inconnuë à nos péres' et dont la manière prend précisément pour guide le style coupé propre au siècle de Sénèque et non plus 'la saine Antiquité. ${ }^{14}$ C'est Diderot qui, dans son Essai de 1778 sur les règnes de Claude et Néron, semble répondre à cette prévention en observant 'que les ennemis de nos philosophes ressemblent quelquefois merveilleusement aux détracteurs de Sénèque', pour mieux faire l'éloge de la manière 
d'éloquence 'précise, vive, énergique, serrée' que celui-ci pratiquait. ${ }^{15}$ Un savant, rapporte Diderot,

me semble avoir mieux caractérisé le style de Sénèque, lorsqu'il a dit de cet auteur qu'il avait de l'abondance avec brièveté, abundantiam in brevitate, et de la véhémence avec facilité. ${ }^{16}$

Jusque dans ses derniers textes, c'est précisément cette abundantia in brevitate que Diderot a placée au cœur de sa réflexion sur l'éloquence. Toute l'enquête épistémologique à laquelle il se livre dans la Lettre sur la simultanéité des sensations s'inscrit même dans la discussion de ce problème rhétorique, au point de culminer dans un long développement consacré à ce qu'il appelle la 'peinture hiéroglyphique' $(L S M, 172)$ des poètes .

'Hiéroglyphe': le terme désigne une sorte d'agglomération de sens propre à représenter plusieurs choses au moyen d'une seule et unique figure. On conçoit quel profit on peut tirer de ce terme dans un contexte où il s'agit de comprendre en quoi la poésie parvient, en un seul trait, à faire voir et entendre, juger et imaginer - bref, à entasser sensations, idées, expressions à la faveur de quelque figure concise et susceptible de peindre 'l'état d'une âme' qui éprouve tout en un moment unique. Au reste, la critique contemporaine n'a pas manqué de relever la vogue dont jouissait alors la notion de hiéroglyphe, rappelant avec constance les noms de Condillac ou encore du théologien anglais William Warburton. ${ }^{17}$ Si rien ne semble mieux assuré que ces remarques, on observera toutefois que l'idée même de hiéroglyphe appartient aussi au patrimoine commun des rhétoriques de l'âge classique. Hérité de travaux sur les pictogrammes de l'Égypte ancienne qui furent menés par les humanistes de la Renaissance et auxquels Diderot fait allusion dans sa Lettre (LSM, 177), l'intérêt pour les hiéroglyphes se limita d'abord, il est vrai, au seul domaine d'une érudition soucieuse de retracer une Prisca Theologia ou, si l'on préfère, une théologie primordiale et mystérieuse dont la sagesse des anciens Grecs et Romains n'aurait été que l'écho affaibli. A partir du XVIIe siècle cependant, ce qui restera de mystère dans le hiéroglyphe en arrivera bientôt à ne désigner que la brièveté allusive des images courtes et vives auxquelles recourt la concision du style. ${ }^{18} \mathrm{C}^{\prime}$ est sous cette forme oratoire et mondaine qu'on le retrouve aussi bien dans le De sapientia veterum de Francis Bacon, traduit en français dès 1619 par Jean Baudoin, que chez Pierre-Daniel Huet. ${ }^{19}$ Paru en 1670, son Traité de l'origine des romans analyse le langage hiéroglyphique des fables mythologiques, de manière à mettre en évidence le laconisme souvent énigmatique des figures oratoires qu'illustre la vivacité du style propre à la fiction. Enfin, comment douter de ce destin littéraire et oratoire à la lecture, par 
exemple, d'un roman licencieux comme L'École des filles, alors que les plaisirs de la conversation entre deux jeunes femmes suggèrent à l'une d'elles l'évocation de cette 'vive peinture' qui, 'dans le murmure et la douce union de deux langues qui se chatouillent', fait en sorte que 'tous les mots dont [les amants] se servent sont autant de mots hiéroglyphiques dont chacun [...] porte une sentence entière? ${ }^{20}$ Autant qu'à Warburton ou qu'à Condillac, c'est donc à toute cette tradition oratoire et mondaine qu'il faut songer lorsqu'en 1751 Diderot se réapproprie une notion devenue solidaire, au fil des siècles, d'un 'art de dire' qui, à la faveur de traits brefs portant en raccourci 'une sentence entière', vient si bien servir l'idéal d'une abundantia in brevitate.

Surtout, le hiéroglyphe se signale par son caractère éminemment pictural qui en fait, suivant l'expression du chevalier de Jaucourt dans l'article 'Hiéroglyphe' de l'Encyclopédie, une véritable 'écriture en peinture.' La Lettre, on l'a vu, parle volontiers de 'peinture hiéroglyphique' $(L S M, 172)$ et, comme l'a déjà montré Jacques Proust, les lectures de Diderot à cette époque ne pouvaient que renforcer cette couleur picturale, qu'il s'agisse du De arte graphica (1668) de Dufresnoy, du Cours de peinture par principes (1708) de Roger de Piles ou encore des Conférences de Charles Le Brun. ${ }^{21}$ Or toute peinture ' $n$ 'a qu'un instant', comme le rappellera plus tard Diderot dans ses Essais sur la peinture (1765) à la suite d'une longue tradition. ${ }^{22}$ Le peintre, assurait déjà Roger de Piles, 'ne peut représenter dans un même tableau que ce qui se voit d'un coup d'œil dans la nature. ${ }^{23}$ Charles Le Brun insistait à son tour sur le fait que le punctum temporis ou, si l'on préfère, 'l'instant unique' que le pinceau doit fixer et représenter renferme une multitude de moments distincts rassemblés en un seul instant où passé et présent s'abolissent:

Le peintre n'ayant qu'un instant dans lequel il doit prendre la chose qu'il veut figurer pour représenter ce qui s'est passé dans ce moment-là, il est quelquefois nécessaire qu'il joigne ensemble beaucoup d'incidents qui aient précédé, afin de faire comprendre le sujet qu'il expose. ${ }^{24}$

Rappelé par tous les traités consacrés à la peinture, ce principe pictural de 'l'instant unique' ne pouvait qu' inviter fortement à rattacher la notion de 'peinture hiéroglyphique' à celui d'un punctum temporis où se retrace le 'tableau mouvant' d'un esprit occupé en un même moment à une multitude de sensations et d'idées.

Punctum temporis, hiéroglyphe, abundantia in brevitate: voilà trois traits essentiels qui constituent autant de points d'ancrage de la réflexion de Diderot dans une tradition à la fois classique, oratoire et picturale dont celui-ci réunit les éléments épars à la faveur des principes propres à la philosophie nouvelle. C'est dire à quel point la 'rhétorique sensualiste' 
qui résulte de ce parcours et dont la Lettre offre une première formulation tire sa force et son originalité de cette étroite liaison entre une tradition oratoire 'ancienne' et une tradition philosophique 'moderne' dont il convient moins, en cette circonstance, de marquer les différences que de souligner la fécondité de leur rencontre. Qu'il s'agisse du hiéroglyphe ou encore de l'abundantia in brevitate, on a tôt fait de s'apercevoir que ces notions n'en appellent plus tant au docere (instruire) au movere (émouvoir) ou au delectare (plaire), triade traditionnelle des officia de l'orateur, qu'à une expérience vécue du temps qui fonde l'éloquence sur la vitalité, la promptitude et l'énergie propres au concours tumultueux des sensations se pressant simultanément dans l'âme. Suivant un mouvement inverse, l'inscription de la tradition rhétorique au sein de la philosophie nouvelle offre, dans la Lettre, l'exemple précieux d'une 'modernité' qui parvient à s'inventer sans pour autant s'élever sur les ruines d'une tradition ou, pour mieux dire, d'une mémoire. Il en résulte un questionnement inouii jusque-là ou, du moins, ignoré d'un empirisme à l'anglaise qui, rêvant depuis Locke d'une transparence parfaite entre les mots et les choses, se plaisait volontiers à faire de la rhétorique une nouvelle 'folle du logis' et de l'analyse des sensations le fondement d'une sémiotique destinée à fixer le sens propre de chaque signe. A la différence de cet empirisme dont il procède, le sensualisme français, du moins si l'on en juge par la Lettre, invente au contraire une sorte de rhétorique des Lumières. C'est ce dont témoigne l'œuvre ultérieure de Diderot, depuis l'éloquence 'sauvage' et énergique de la harangue du vieillard dans le Supplément au voyage de Bougainville et le ton véhément de ces appels à la révolte dans l'Histoire des deux Indes de l'abbé Raynal jusqu'à cette peinture vive et serrée d'impressions simultanées que réclament les Essais sur la peinture:

Montre-moi Commode [écrit Diderot à l'intention de l'artiste] abandonné aux bêtes. Que je le voie sur ta toile déchiré à coups de crocs. Fais-moi entendre les cris mêlés de la fureur et de la joie autour de son cadavre. ${ }^{25}$

Cette dissémination ultérieure de traits où seront appelées à s'effacer les références explicites à la rhétorique procède pourtant d'un effort initial de théorisation où certaines notions issues de la tradition oratoire trouvaient leur fondement dans l'énergie et la vitalité de la sensation, laquelle s'élevait en retour jusqu'aux arts du discours. Diderot, poète de l'énergie, écrivait Jacques Chouillet: ${ }^{26}$ sans nul doute, mais dans la mesure où une méditation sur la temporalité s'allie chez lui à un art oratoire qui se saisit du tableau mouvant de l'âme à la pointe de quelque figure hiéroglyphique, entremêlant dès lors sensations et idées un peu à la 
manière dont les langues libertines s'entortillent et s'enlacent dans l'École des filles.

\author{
MARC-ANDRÉ BERNIER \\ Université du Québec à Trois-Rivières
}

\title{
Notes
}

1 Voir, en particulier, les annotations de la Lettre sur les sourds et muets dans l'édition qu'en a donnée Paul Hugo Meyer dans Diderot Studies 7 (Genève: Librairie Droz, 1965); et Marian Hobson, 'Quelques références dans la Lettre sur les sourds et muets', Diderot Studies 18 (Genève: Librairie Droz, 1975) 111-19.

2 Boileau, Le lutrin, chant II, v. 164; cité par Diderot, Lettre sur les sourds et muets à l'usage de ceux qui entendent et qui parlent, 1751, CEuvres complètes, tome IV (Paris: Hermann, 1978) 169. Toutes les références, qui renvoient à cette édition établie et présentée par Jacques Chouillet, seront notées ainsi dans la suite du texte: (LSM, 169).

3 Louis Le Laboureur, Les avantages de la langue françoise sur la langue latine (Paris: Florentin Lambert, 1667) 23.

4 Bernard Tocane, 'Ordre naturel des mots et pensée naturelle', L'idée de nature en France dans la seconde moitié $d u$ XVIIe siècle (Paris: Klincksieck, 1978) 378-389; Ulrich Ricken. 'Die Kontroverse Du Marsais und Beauzée gegen Batteux, Condillac und Diderot - Ein Kapitel der Auseinandersetzung zwischen Sensualismus und Rationalismus in der Sprachdiskussion der Aufklärung', History of Linguistic Thought and Contemporary Linguistics (Berlin / New-York: Walter de Gruyter, 1976). A juste titre, ce dernier rappelle 'die philosophische Aktualität der Problematik, daß innerhalb weniger Jahre drei wichtige Schriften erschienen, die im Gegensatz zu Du Marsais die Begründung einer sensualistischen Wortstellungstheorie unternehmen' (465) ['l'actualité philosophique de la problématique, alors qu'en l'espace de quelques années paraissent trois textes importants qui, s'opposant à Dumarsais, entreprennent de fonder une théorie sensualiste de l'ordre syntaxique' (nous traduisons)].

5 Article 'Inversion' (rédigé entre 1751 et 1756), Encyclopédie, ou dictionnaire raisonné des sciences, des arts et des métiers, tome 8; repris dans Les véritables principes de la grammaire, et autres textes (Paris: Fayard, 1987) 85-86. Pour une discussion précise de cette thèse, voir Sylvain Auroux, 'Construction et inversion', La sémiotique des Encyclopédistes (Paris: Payot, 1979) 191-212.

6 Charles Batteux, 'Que l'arrangement naturel des mots est réglé par l'importance des objets', Principes de la littérature, 1748 (Paris: Desaint \& Saillant, 1775) première section, 4-5, 7-8. Sur cette même question, voir aussi Condillac, 'Essai sur l'origine des connoissances humaines', CEuvres philosophiques de Condillac, vol. 1 (Paris: PUF, 1947) §117-§124, 92 sq. 
7 Comparez LSM, 156 avec 'Inversion' (loc. cit. 75) et avec Principes de la littérature (op. cit. 10 sq.), alors qu' on retrouve à chaque fois un commentaire de l'inversion à laquelle donne lieu le début du Pro Marcello de Cicéron.

8 Sur ces distinctions, voir également les 'Observations sur l'extrait que le journaliste de Trévoux a fait de la Lettre sur les Sourds et Muets' (LSM, 214): 'L'ordre de notre syntaxe est didactique', précise alors Diderot en réponse à un article paru dans le Journal de Trévoux et qui confondait 'ordre didactique' et 'ordre d'institution.'

9 Il s'agit là d'une thèse qui, on le sait, ne manquera pas d'être reprise pendant tout le XVIIIe siècle. On consultera par exemple Rivarol pour lequel 'c'est en vain que les passions nous bouleversent et nous sollicitent de suivre l'ordre des sensations [...]. Ce qui n'est pas clair n'est pas français, ce qui n'est pas clair est encore anglais, italien, grec ou latin'; L'universalité de la langue française, 1784, De l'universalité européenne de la langue française (Paris: Fayard, 1995) 162.

10 'La prise en compte, chez Diderot, d'une perspective historique, conduit à une thèse plutôt géniale. ... A la place des catégories d'une raison intemporelle se retrouvent des catégories qui résultent de la transformation de la sensation en réflexion' (je traduis); Ulrich Ricken, 'Die Kontroverse Du Marsais und Beauzée gegen Batteux, Condillac und Diderot', loc. cit. 467.

11 Abbé Goujet, 'Écrits François sur l'Eloquence en général', Bibliothèque françoise ou Histoire de la littérature françoise, tome II, chap. III (Paris, 1740) 53.

12 Discours sur les spectacles (Paris: Coignard Fils, 1733) 13.

13 Cité par l'abbé Desfontaines dans L'esprit de l'abbé Desfontaines, tome II (Paris, 1757) 90. Sur Porée et sur la vogue du 'stile coupé', voir Claude Buffier, qui oppose 'les panégyriques de Cicéron, du P. Bourdaloue \& du P. Cossart' à ceux 'trop brillans de Pline le Jeune, de M. Fléchier \& du P. Porée' dans son 'Traité philosophique et pratique d'éloquence', Cours de science (Paris: G. Cavelier, 1732) 309.

14 'Discours sur l'éloquence', Philippiques de Démosthéne et Catilinaires de Cicéron traduites par Monsieur l'Abbé d'Olivet (Paris: Gaudoin, 1736) 19.

15 Denis Diderot, Essai sur les règnes de Claude et Néron et sur la vie et les écrits de Sénèque, pour servir d'introduction à la lecture de ce philosophe, CEuvres complètes de Diderot, tome III (Paris: Garnier Frères, 1875) 179, 195-196. Malgré ces témoignages, le rôle essentiel que joue, à partir de la Régence, la figure de Sénèque en tant que 'symbole capable de caractériser toute une orientation littéraire', n'a été évoqué de nos jours que par Basil Munteanu: voir 'Quintilien dans la "Querelle". Les "nouveaux Sénèques" et le "retour à l'antique"'; Constantes dialectiques en littérature et en histoire (Paris: Didier, 1967) 180-83.

16 Ibid., 235.

17 Voir aussi bien Paul Hugo Meyer ('Introduction', Diderot Studies, op. cit. 17) que Jacques Chouillet ('Introduction' à la Lettre sur les sourds et muets, op. cit. 116) et Jean Starobinski dans son édition de l'Essai sur l'origine des langues (Paris: Gallimard, 1990) 73, note 1.

18 Sur l'absorption de la notion de hiéroglyphe par les 'arts de dire' et 'par le goût des "mondains" pour un ingenium plus rapide', voir Marc Fumaroli, 'Hiéroglyphes et Lettres: la "sagesse mystérieuse des Anciens" au XVIIe siècle', XVIIe siècle 158, (janvier-mars 1988) 14 sq. 
19 Jean Baudoin, La Sagesse mystérieuse des Anciens, ombragée du voile des Fables, appliquées moralement aux secrets de l'État et de la Nature (Paris: Julliot, 1619); sur ce texte et sur Huet, voir Fumaroli, loc. cit. 15-19.

20 L'École des Filles ou la Philosophie des Dames. Divisée en deux dialogues: agere et pati. Corrigé et augmenté d'un combat du Vit et du Con, 1668, L'Enfer de la Bibliothèque nationale. CEuvres érotiques du XVIIe siècle, vol. 7 (Paris: Fayard, 1985) 257.

21 Voir Jacques Proust, 'L'initiation artistique de Diderot', Gazette des Beaux-Arts, 102e année, 6e période, LV (avril 1960) 225-32.

22 Diderot, Essais sur la peinture, CEuvres. Esthétique-Théâtre, tome IV (Paris: Laffont, 1996) 496; édition établie par L. Versini.

23 Roger de Piles, Cours de peinture par principes (Paris: Gallimard, 1989) 36.

24 'Conférence de Charles Le Brun sur les Israélites recueillant la manne au désert, par Poussin', 1667, L'expression des passions et autres conférences (Paris: Éditions Dédale, Maisonneuve et Larose, 1994) 185.

25 Diderot, 'Essais sur la peinture' op. cit. 501 (nous soulignons).

26 Voir Jacques Chouillet, Diderot, poète de l'énergie (Paris: PUF, 1984). 